\title{
Quarantine Barriers and Facilitators in COVID-19 Pandemic: Short Communication
}

Ghonoodi. $\mathrm{F}^{1}$

Mohammadnejad. E2

Ehsani. SR ${ }^{3}$

${ }^{*}$ Salehi. $Z^{4}$

1- MSc of Nursing, Faculty of Nursing and Midwifery, Tehran University of Medical Sciences, Tehran, Iran.

2- Ph.D. in Nursing, Assistant Professor, Nursing Department, Faculty of Nursing and Midwifery, Tehran University of Medical Sciences, Tehran, Iran.

3- Nursing Office, Imam Khomeini Clinical and Hospital Complex, Tehran University of Medical Sciences, Tehran, Iran.

4- ( ${ }^{*}$ Corresponding Author) MSc of Nursing, Faculty of Nursing and Midwifery, Iran University of Medical Sciences, Tehran, Iran.

Email: salehi.z2018@gmail.com

\section{Abstract}

Introduction: COVID-19 is a disease caused by a novel type of coronavirus with no definitive treatment and vaccine. Quarantine is known to be one of the oldest and most effective tools for controlling the spread of infectious diseases.

Objective: This study aimed at determining the Quarantine Barriers and Facilitators in COVID-19 pandemic.

Materials and Method: In this review, PubMed, Scopus, Web of Science (WoS), Magiran and SID databases were used to search for articles with keywords such as, COVID-19, Isolation, Quarantine, Pandemic.

Discussion and Conclusion: This study showed that the most important deterrent in implementing quarantine is the lack of knowledge and financial problems and the most basic factor facilitating the implementation of quarantine is increasing awareness of the people of the community about quarantine. For better implementation, socio-economic support incentives and strengthening information and educational platforms are recommended.

Keywords: COVID19, Pandemic, Quarantine. 


\section{موانع و تسهيل كنندههاى قرنطينه در باندميك كوويد 19: يك مقاله كوتاه}

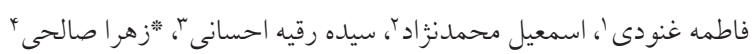

جكيده

مقدمه: كوويد-19 بيمارى است كه با نوع جديد ويروس كرونا ايجاد شده است كه فاقد درمان و واكسن مىباشد. قرنطينه به عنوان يكى از قديمىترين و مؤثرترين ابزارهاى كنترل شيوع بيمارىهاى واگير و عفونى شناخته مىشود. هدف: هدف از اين مطالعه بررسى موانع و تسهيل كنندهاى قرنطينه در پاندميك كوويد 9 استر مواد و روشها: در اين مطالعه جستجو با استفاده از بايخاههاى اطلاعاتى ملى Scientific Information Database (SID)

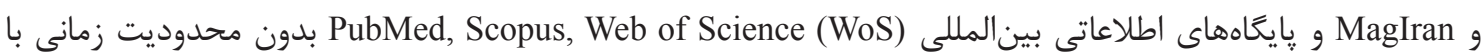
كليدوازههاى Pandemic, COVID-19, Isolation, Quarantine و تركيب اين وازهها جستجو شد. بحث و نتيجهَيرى: اين مطالعه نشان داد مههمترين دليل بازدارنده در اجراى قرنطينه كمبود دانش و آكاهى و مشكلات

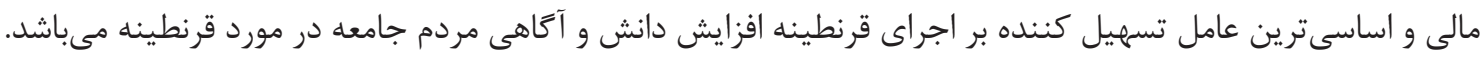

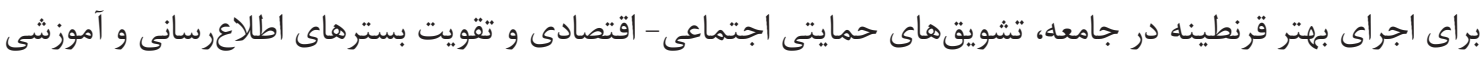
ريشنهاد مىشود.

كلمات كليدى: قرنطينه، پياندمى، كوويد-19

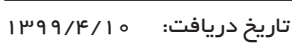

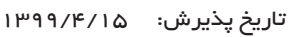

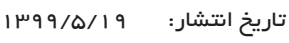

كروناويروس جديد را ششمين عامل وضعيت اضطرارى بهداشت مقدمه عمومى در سرتاسر جهان اعلام نمود كه به عنوان تهديدى براى بيمارى كروناويروس (COVID-19) يك بيمارى عفونى است كه تمام كشورها به شمار مىرود (9).

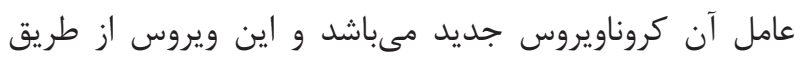

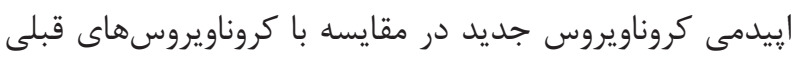
قطرات بزاق يا ترشحات بينى به هنحام سرفه يا عطسه، كَسترش شايع در انسان كستردهتر بوده كه اين موضوع بيانكر قدرت سرايت

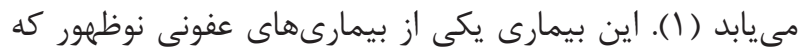

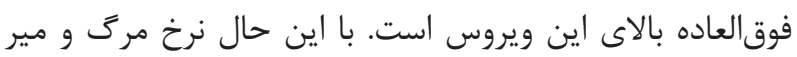
باعث نكرانى شديد در سرتاسر جهان شده و يك تهديد جدى لئى

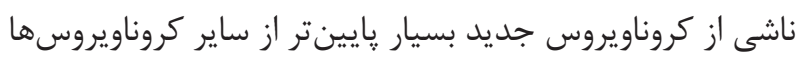

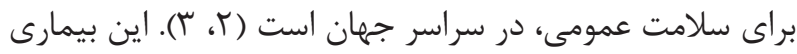
نظير سارس (SARS)، مرس (MERS) و ويروس هاى ديخرى نظير بروسي (SARS: جديد ويروسى، پس از سندرم شديد تنفسى حاد

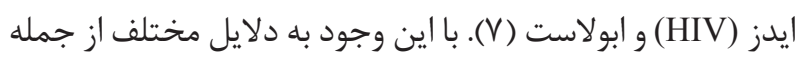
Severe Acute Respiratory Syndrome)

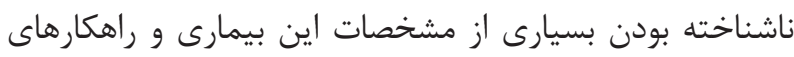
خاورميانه (Middle East Respiratory Syndrome: MERS) مقابله با آن، تنوع بالاى علائم، عفونت شديد، طولانى بودن دوران نهفتگى و همجنين قدرت و گسترش سريع انتشار بالاى آن، تأثير سومين بيمارى همه گير ويروسى در قرن آ است (Y)، ه). سازمان

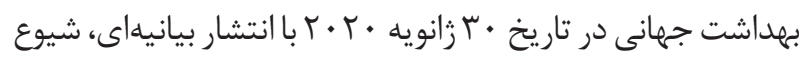


زمانى با كليدوازههاى، Pandemic به مقالات بيشتر، جستجو به صورت رو به عقب (بررسى ليست

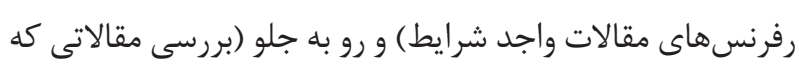
در مطالعات واجد شرايط به آنها استناد شده بود) نيز انجام شد.

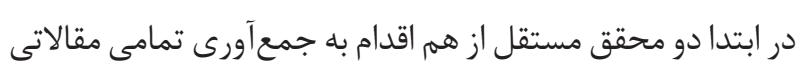

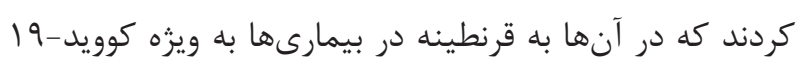

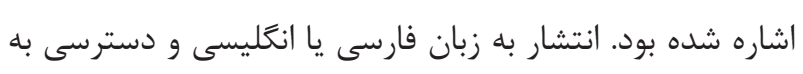
متن كامل مقالات جزء معيارهاى ورود به مطالعه بود. مطالعات غير مرتبط با موضوع و متون خاكسترى از مطالعه خارج شدند. براى اينكه سوَرايى به حداقل برسد جستجوى مقالات، انتخاب مطالعات، ارزيابى كيفيت متدولوزى مقالات و استخراج دادهها

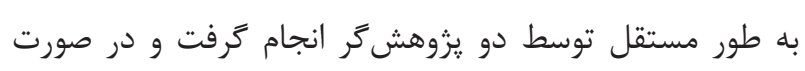

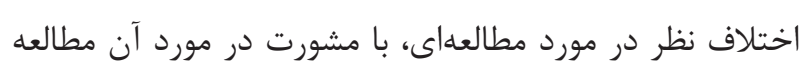

$$
\text { تصميم خيرى مىشد. }
$$

يافته ها در جستجوى اوليه يايكاههاى ملى و بينالمللى لو و مقاله يافت شد. در مرحله غربالكرى V9 مقاله غير مرتبط از تحليل خارج شدند.

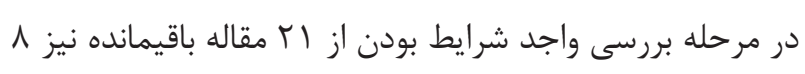
مقاله به دليل عدم كزارش درست و يا وجود داده يرت إز از تحليل خارج شد. در نهايت با 1 مقاله وارد تحليل شد. مقالات بات باقيمانده مورد خلاصهسازى قرار ترفتند (جدول ()).

\section{بحث و نتيجهَيرى}

اين مطالعه مرورى با هدف بررسى موانع و تسهيل كنندههاى بندي

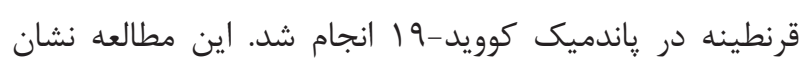
داد برخى از عوامل نقش تسهيل كننده و برخى عوامل نقش دانش

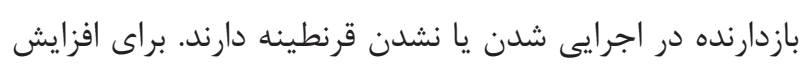

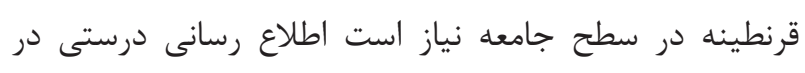

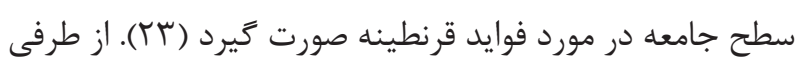
تشويقهاى حمايتى اقتصادى در نظر كرفت و مايحتاج آنها را ران

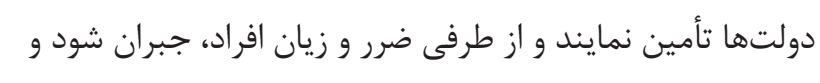

بسيار زيادى بر سلامت روانى عموم مردم نكرانى فزايندهاى نسبت

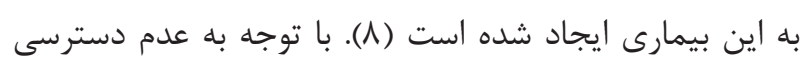

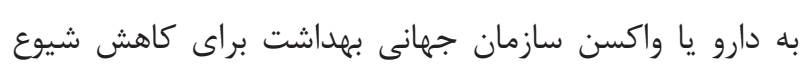

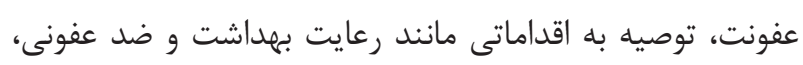

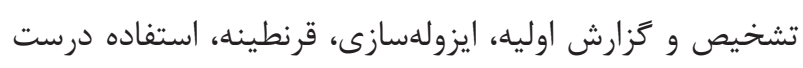
و منطقى از تجهيزات حفاظتى شخصى، فاصله گيرى اجتماعى و محدويت مسافرت را توصيه كرده است (9). قرنطينه به عنوان

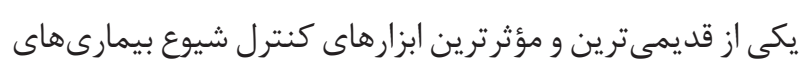
واخير هنگامى استفاده مىشود كه شخص علائم بيمارى دارد

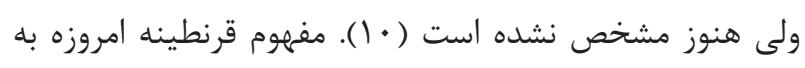

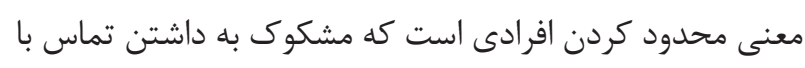

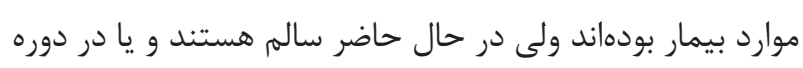

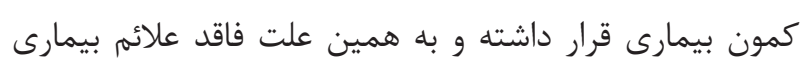

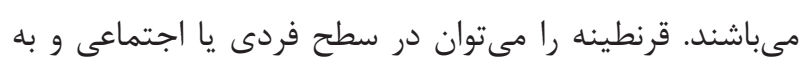

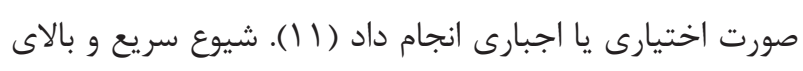

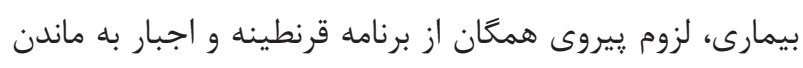

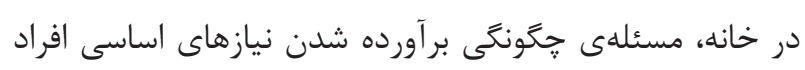

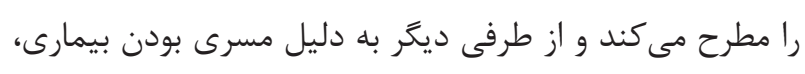

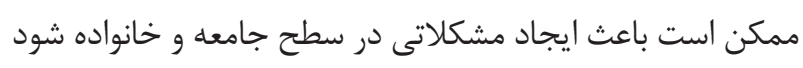

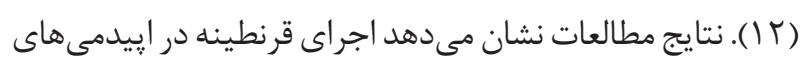
بزرى در مواردى كه مردم از آن حمايت نمى كنند كمتر است (^).

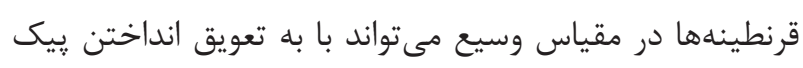
اييدمى به سيستم خدمات درمانى فرصت دهد تا بتواند در برابر

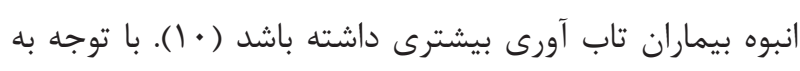

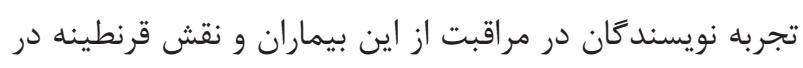
كنترل و ييشخيرى از بيمارى ها، اين مطالعه با هدف بررسى موانع

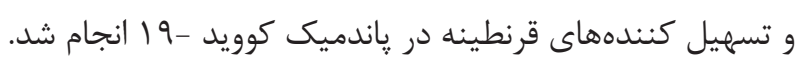

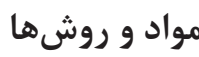

در اين مطالعه مرورى با هدف بررسى موانع و تسهيل كنندههاى

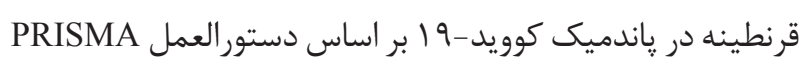

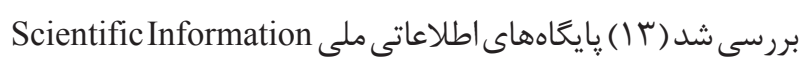
و Matabase (SID) PubMed, Scopus, Web of Science (WoS) 
جدول ا- خلاصهسازى مقالات مرتبط با قرنطينه در ياندميك كوويد-19

\begin{tabular}{|c|c|}
\hline دلايل اصلى & آيتم \\
\hline 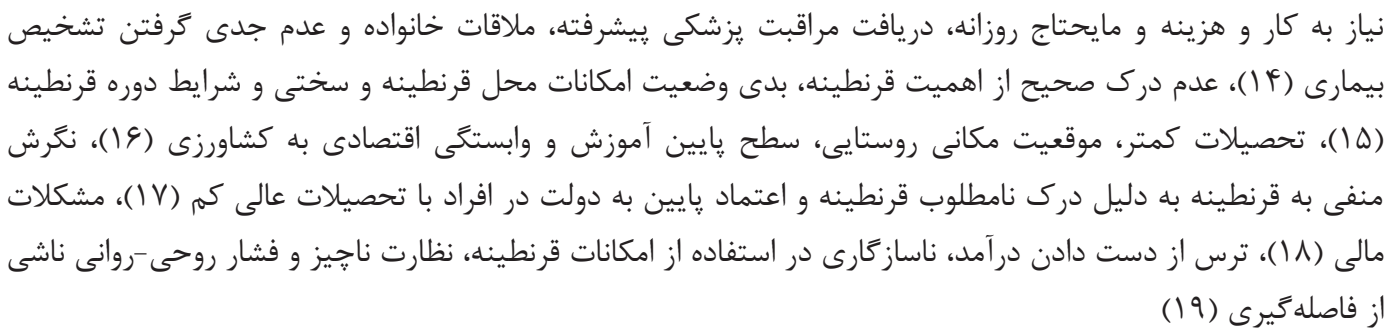 & اجراى قرنطينه بازدارنده در \\
\hline 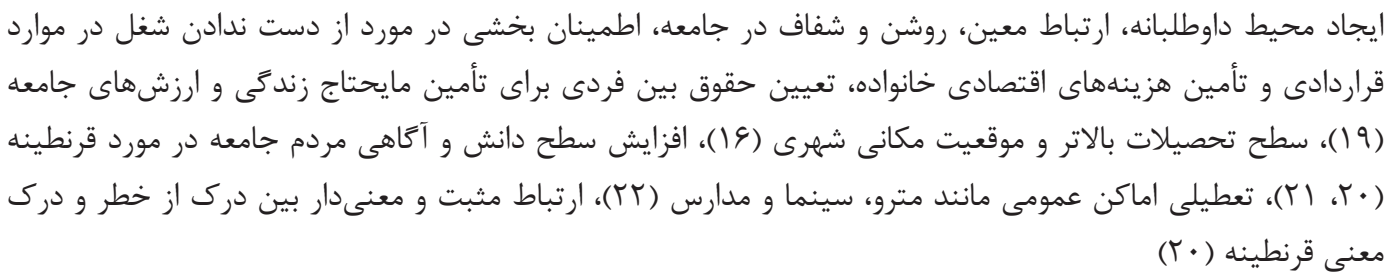 & در اجراى قرنيل كنينه \\
\hline
\end{tabular}

از زَزينههاى مناسب مبارزه با بيمارى، قرنطينه فورى كانونهاى

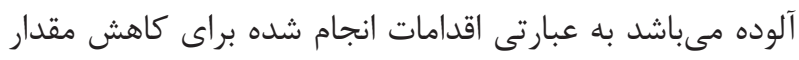

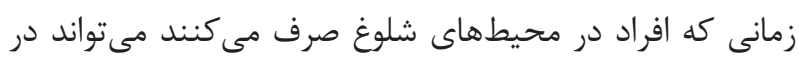

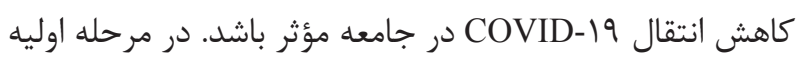
شيوع، استراتزى اصلى تمركز بر جلو گيرى از انتشار آلودىى اوليه،

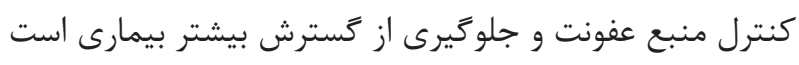

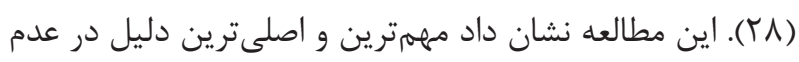

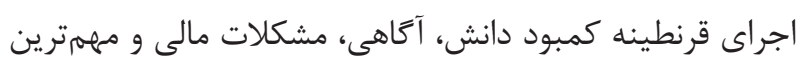

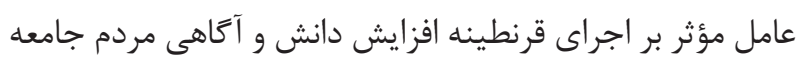

$$
\text { در مورد قرنطينه مىباشد. }
$$

\section{References}

1- Who. Coronavirus 2020 Available from: https://www.who.int/ health-topics/coronavirus\#tab=tab_1.

2- Jernigan DB, Team CC-R. Update: Public health response to the coronavirus disease 2019 outbreak - United States, February 24, 2020. MMWR Morb Mortal Wkly Rep. 2020; 69(8): 216-9. http:// dx.doi.org/10.15585/mmwr.mm6908e1 www.ncbi.nlm.nih.gov/ pubmed/32106216

3- Liao X, Wang B, Kang Y. Novel coronavirus infection during the 2019-2020 epidemic: Preparing intensive care units-the experience in Sichuan Province, China. Intensive Care Med. 2020;46(2):35760. http://dx.doi.org/10.1007/s00134-020-05954-2 www.ncbi. nlm.nih.gov/pubmed/32025779

4- Da Costa VG, Moreli ML, Saivish MV. The emergence of SARS, MERS and novel SARS-2 coronaviruses in the 21 st century. Arch
و همكاران در ليبريا نشان داد يكى از ييامدهاى (Nyenswah)

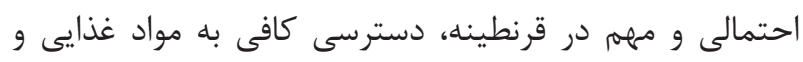

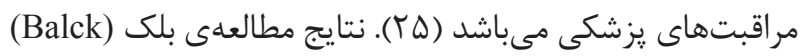

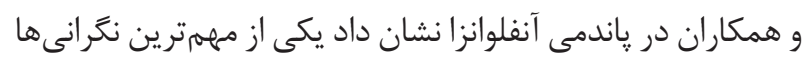

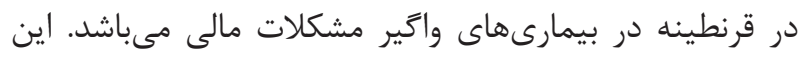

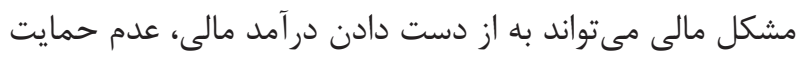

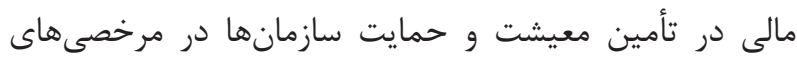

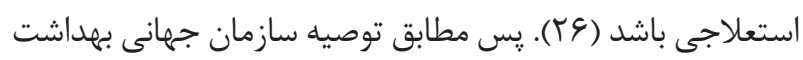
براى بيمارىهايى كه واكسن و درمان ندارند و بسيار مسرى نيز

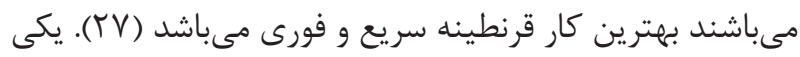

Virol. 2020;165(7):1517-26. http://dx.doi.org/10.1007/s00705020-04628-0 www.ncbi.nlm.nih.gov/pubmed/32322993

5- Gorbalenya AE, Baker SC, Baric RS, de Groot RJ, Drosten C, Gulyaeva AA, et al. 2020. http://dx.doi. org/10.1101/2020.02.07.937862

6- Lai CC, Shih TP, Ko WC, Tang HJ, Hsueh PR. Severe acute respiratory syndrome coronavirus 2 (SARS-CoV-2) and coronavirus disease-2019 (COVID-19): The epidemic and the challenges. Int J Antimicrob Agents. 2020;55(3):105924. http:// dx.doi.org/10.1016/j.ijantimicag.2020.105924 www.ncbi.nlm.nih. gov/pubmed/32081636

7- Tavakoli A, Vahdat K, Keshavarz M. Novel Coronavirus Disease 2019 (COVID-19): An Emerging Infectious Disease in the 21st Century. Iranian South Med J. 2020;22(6):432-50. http://dx.doi. 
org/10.29252/ismj.22.6.432

8- Gao J, Zheng P, Jia Y, Chen H, Mao Y, Chen S, et al. Mental health problems and social media exposure during COVID-19 outbreak. PLoS One. 2020;15(4):e0231924. http://dx.doi.org/10.1371/ journal.pone.0231924 www.ncbi.nlm.nih.gov/pubmed/32298385

9- WHO. COVID-19 Response 2020. Available from: https://www. who.int/home.

10- Wilder-Smith A, Freedman DO. Isolation, quarantine, social distancing and community containment: pivotal role for old-style public health measures in the novel coronavirus (2019-nCoV) outbreak. J Travel Med. 2020;27(2). http://dx.doi.org/10.1093/jtm/ taaa020 www.ncbi.nlm.nih.gov/pubmed/32052841

11- Afrashteh S, Alimohamadi Y, Sepandi M. The Role of Isolation, Quarantine and Social Distancing in Controlling the COVID-19 Epidemic. J Mil Med. 2020;22(2):210-1.

12- Chang HY, Chiou CJ, Chen NS. Impact of mental health and caregiver burden on family caregivers' physical health. Arch Gerontol Geriatr. 2010;50(3):267-71. http://dx.doi.org/10.1016/j. archger.2009.04.006 www.ncbi.nlm.nih.gov/pubmed/19443058

13- Ades AE, Lu G, Higgins JP. The interpretation of randomeffects meta-analysis in decision models. Med Decis Making. 2005;25(6):646-54.http://dx.doi.org/10.1177/0272989X05282643 www.ncbi.nlm.nih.gov/pubmed/16282215

14- Teh B, Olsen K, Black J, Cheng AC, Aboltins C, Bull K, et al. Impact of swine influenza and quarantine measures on patients and households during the H1N1/09 pandemic. Scand J Infect Dis. 2012;44(4):289-96. http://dx.doi.org/10.3109/00365548.2011.631 572 www.ncbi.nlm.nih.gov/pubmed/22106922

15- Nadim M. Consideration of Quarantine Conditions in Bushehr Between 1905 and 1915, Based on Baliuz of Britain Reports. J Res Hist Med. 2013;2(1):26-34.

16- Edirne T, Avci DK, Dagkara B, Aslan M. Knowledge and anticipated attitudes of the community about bird flu outbreak in Turkey, 2007-2008: a survey-based descriptive study. Int J Public Health. 2011;56(2):163-8. http://dx.doi.org/10.1007/s00038-0100131-x www.ncbi.nlm.nih.gov/pubmed/20217176

17- Kim EY, Liao Q, Yu ES, Kim JH, Yoon SW, Lam WW, et al. Middle East respiratory syndrome in South Korea during 2015: Riskrelated perceptions and quarantine attitudes. Am J Infect Control. 2016;44(11):1414-6. http://dx.doi.org/10.1016/j.ajic.2016.03.014 www.ncbi.nlm.nih.gov/pubmed/27130900

18- Kavanagh AM, Mason KE, Bentley RJ, Studdert DM, McVernon J, Fielding JE, et al. Leave entitlements, time off work and the household financial impacts of quarantine compliance during an H1N1 outbreak. BMC Infect Dis. 2012;12:311. http:// dx.doi.org/10.1186/1471-2334-12-311 www.ncbi.nlm.nih.gov/ pubmed/23164090
19- DiGiovanni C, Conley J, Chiu D, Zaborski J. Factors influencing compliance with quarantine in Toronto during the 2003 SARS outbreak. Biosecur Bioterror. 2004;2(4):265-72. http:// dx.doi.org/10.1089/bsp.2004.2.265 www.ncbi.nlm.nih.gov/ pubmed/15650436

20- Cava MA, Fay KE, Beanlands HJ, McCay EA, Wignall R. Risk perception and compliance with quarantine during the SARS outbreak. J Nurs Scholarsh. 2005;37(4):343-7. http://dx.doi. org/10.1111/j.1547-5069.2005.00059.x www.ncbi.nlm.nih.gov/ pubmed/16396407

21- McVernon J, Mason K, Petrony S, Nathan P, LaMontagne AD, Bentley R, et al. Recommendations for and compliance with social restrictions during implementation of school closures in the early phase of the influenza A (H1N1) 2009 outbreak in Melbourne, Australia. BMC Infect Dis. 2011;11:257. http:// dx.doi.org/10.1186/1471-2334-11-257 www.ncbi.nlm.nih.gov/ pubmed/21958428

22- Pelicioni PHS, Lord SR. COVID-19 will severely impact older people's lives, and in many more ways than you think! Braz J Phys Ther. 2020;24(4):293-4. http://dx.doi.org/10.1016/j. bjpt.2020.04.005 www.ncbi.nlm.nih.gov/pubmed/32387005

23- Tracy CS, Rea E, Upshur RE. Public perceptions of quarantine: community-based telephone survey following an infectious disease outbreak. BMC Public Health. 2009;9:470. http:// dx.doi.org/10.1186/1471-2458-9-470 www.ncbi.nlm.nih.gov/ pubmed/20015400

24- Daniel L. Improving the Delivery of Care to Limited English Proficiency Patients. J Obstet, Gynecol \& Neonatal Nurs. 2015;44:S38-S9. http://dx.doi.org/10.1111/1552-6909.12720

25- Nyenswah T, Blackley DJ, Freeman T, Lindblade KA, Arzoaquoi SK, Mott JA, et al. Community quarantine to interrupt Ebola virus transmission - Mawah Village, Bong County, Liberia, AugustOctober, 2014. MMWR Morb Mortal Wkly Rep. 2015;64(7):17982. www.ncbi.nlm.nih.gov/pubmed/25719679

26- Blake KD, Blendon RJ, Viswanath K. Employment and compliance with pandemic influenza mitigation recommendations. Emerg Infect Dis. 2010;16(2):212-8. http://dx.doi.org/10.3201/ eid1602.090638 www.ncbi.nlm.nih.gov/pubmed/20113549

27- Fan C, Liu L, Guo W, Yang A, Ye C, Jilili M, et al. Prediction of Epidemic Spread of the 2019 Novel Coronavirus Driven by Spring Festival Transportation in China: A Population-Based Study. Int J Environ Res Public Health. 2020;17(5). http://dx.doi.org/10.3390/ ijerph17051679 www.ncbi.nlm.nih.gov/pubmed/32143519

28- Nutbeam D, McGill B, Premkumar P. Improving health literacy in community populations: a review of progress. Health Promot Int. 2018;33(5):901-11. http://dx.doi.org/10.1093/heapro/dax015 www.ncbi.nlm.nih.gov/pubmed/28369557 